\title{
SEMICONDUCTOR OPTOELECTRONICS \\ Experimental investigation of polarisation rotation in semiconductor optical amplifiers
}

\author{
B.F. Kennedy, S. Philippe, P. Landais, A.L. Bradley and H. Soto
}

\begin{abstract}
An experimental study of polarisation rotation in a semiconductor optical amplifier is presented. Two techniques are used to investigate the gain and birefringence along the two eigenmodes of the component waveguide, with and without injection. The first investigation is based on the residual reflectivity of the facet mirrors. From the modulation depth of polarisation resolved spectra, the gain and the refractive indices of these modes are determined. The second investigation takes into account the variation of the eigenmode gain and refractive indices as a function of injected power over the range 83.3 to $413 \mu \mathrm{W}$. The results of these two experiments are compared, and it is determined that the refractive index and the single-pass gain along the horizontal axis of the waveguide are higher than those along the vertical axis. Both gain and refractive index differences increase as a function of injected power in the sample under test. Furthermore, it is concluded that polarisation rotation in this component is heavily influenced by the power dependence of the relative gain in the TE and TM modes and birefringence.
\end{abstract}

\section{Introduction}

To achieve all-optical signal processing, various configurations based on nonlinearities in semiconductor optical amplifiers (SOAs), e.g. crossgain modulation, crossphase modulation and four-wave mixing, have been presented [1-3]. Nonlinear polarisation rotation (NPR) has also been demonstrated [4-7], relating to a switch of the state of polarisation of a beam injected into the device. Thus far, considerable attention has focused on applications which may emerge from the NPR [6-9]. Some groups have looked at the underlying physical origins $[4,8,9]$ but a full characterisation of the polarisation change has yet to be performed, to the best of the authors' knowledge. The aim of this paper is to provide a deeper analysis of the nonlinear polarisation rotation in an SOA by applying two different methods, which probe the polarisation dependent gain and birefringence, with and without signal injection. Measurements of the polarisation state, as achieved using a polarimeter, provide information on the ellipticity of the output signal. Using this novel method, information on the power dependence of the SOA birefringence and TE to TM gain ratio can be extracted, complementing earlier work by Manning et al. [4]. Experimental results are presented and discussed, following which conclusions are drawn.

\section{(C) IEE, 2004}

IEE Proceedings online no. 20040287

doi: 10.1049/ip-opt:20040287

Paper first received 16th June and in revised form 17th December 2003

B.F. Kennedy and P. Landais are with the School of Electronic Engineering, Dublin City University, Dublin 9, Ireland

S. Philippe and A.L. Bradley are with the Physics Department, Trinity College Dublin, Dublin 2, Ireland

H. Soto is with Centro de Investigación Cientifica y de Educación Superio de Ensenada, Km. 107 Carr., Tijuana-Ensenada, Ensenada, Baja California 22860, Mexico

\section{Experimental investigation and discussion}

The SOA under test is a commercially available component. It is a $1.5 \mathrm{~mm}$ long bulk InGaAsP active region surrounded by InP. This structure was grown by MOCVD and it is quoted as having low polarisation dependent gain. Both facets are anti-reflection (AR) coated, with a tilted output of $12^{\circ}$ to reduce the Fabry-Perot resonance due to the residual reflectivity of the facets. For all the experiments the component is temperature regulated at $20^{\circ} \mathrm{C}$. In Fig. 1 the light-output characteristic is presented. Below $220 \mathrm{~mA}$, the SOA acts as an LED; above this current the spontaneous emission is amplified and guided in the active region; the component then acts as an amplifier. In the inset, the emission spectrum is shown at a bias current of $400 \mathrm{~mA}$. It features a peak at $1574 \mathrm{~nm}$ and a $3 \mathrm{~dB}$ linewidth of $27 \mathrm{~nm}$.

We focus on two possible origins of NPR: the gain discrepancy between the eigen-axes of the waveguide, and birefringence due to different values of refractive indices for the modes. In the first experiment, these effects are investigated without injected power, providing information on the underlying properties of the waveguide. In the next experiment they are studied as a function of injected power. Both set-ups are in free space, allowing optimum control and preservation of the state of polarisation of the injected and collected signals.

\subsection{Gain and birefringence in SOA without injection}

The light output is collected by a 0.25 NA AR coated microscope objective; it passes through a $50 \mathrm{~dB}$ extinction ratio polarisation beam splitter (PBS) precisely aligned along the eigenmodes of the SOA waveguide The polarisation resolved amplified spontaneous emission (ASE) spectra are recorded for both eigenmodes using an optical spectrum analyser (HP 86143A) with a resolution of $0.07 \mathrm{~nm}$, as shown in Fig. 2. These eigenmodes are referred to as TE (horizontal) and TM (vertical) throughout this paper. From the ASE modulation depth, introduced by the 


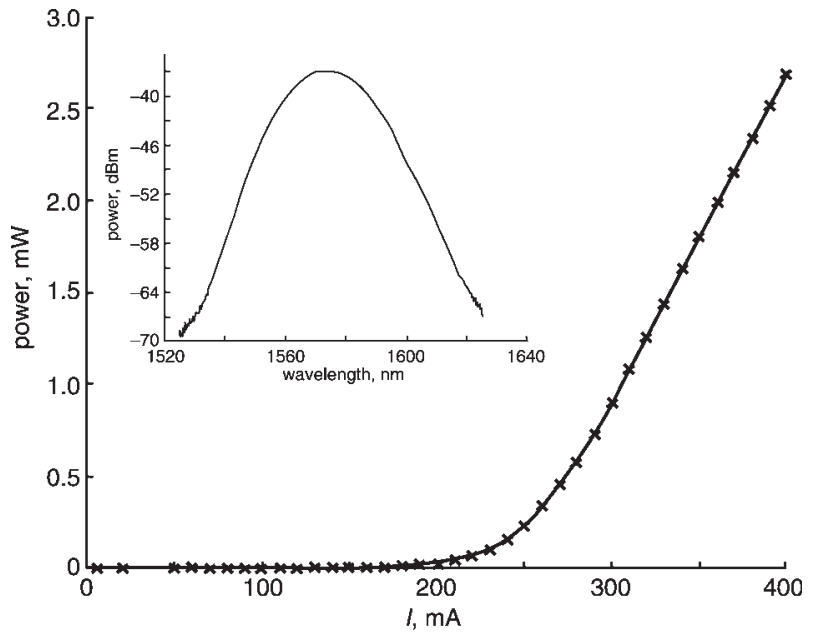

Fig. 1 L/I characteristic of SOA under test Inset: ASE spectrum at $400 \mathrm{~mA}$

Fabry-Perot resonance, we determine the wavelength dependence of the gain $[10,11]$ and the refractive index for TE and TM. The gain reflectivity product spectrum, $\gamma$, is calculated from the formula

$$
\gamma(\lambda)=\frac{\sqrt{r(\lambda)}-1}{\sqrt{r(\lambda)}+1}
$$

where $r$, the modulation depth around the troughs, is given by $\left(P_{\max 1}+P_{\max 2}\right) /\left(2 P_{\min }\right), P_{\max 1}$ and $P_{\max 2}$ represent neighbouring peak modulation power values and $P_{\min }$ the minimum power between these peaks. $\gamma$ is linked to the single-pass gain, $G$, by

$$
\gamma=\sqrt{R_{1}(\lambda) R_{2}(\lambda)} G(\lambda)
$$

where $R_{1}$ and $R_{2}$ are the reflectivities of the output facets. We assume that $R_{1}$ and $R_{2}$ are equal and constant over the spectral range of our study, in accordance with [12]. The single-pass gain is calculated from the TE and TM spectra, as shown in Fig. 2, using (1) and (2). Our investigation is limited to bias currents above $300 \mathrm{~mA}$, as below this current the anti-reflection coatings and waveguide tilting minimise the ripples. Figure 3 shows four TM and TE single-pass gain spectra from $1565 \mathrm{~nm}$ to $1595 \mathrm{~nm}$ for bias currents of $300 \mathrm{~mA}, 350 \mathrm{~mA}, 400 \mathrm{~mA}$ and $450 \mathrm{~mA}$. As the current increases, the peak value of the gain increases for both modes. It is noted that for bias currents from $350 \mathrm{~mA}$ to $450 \mathrm{~mA}$, the maximum single-pass $\mathrm{TE}$ gain and the maximum single-pass TM gain are approximately equal.

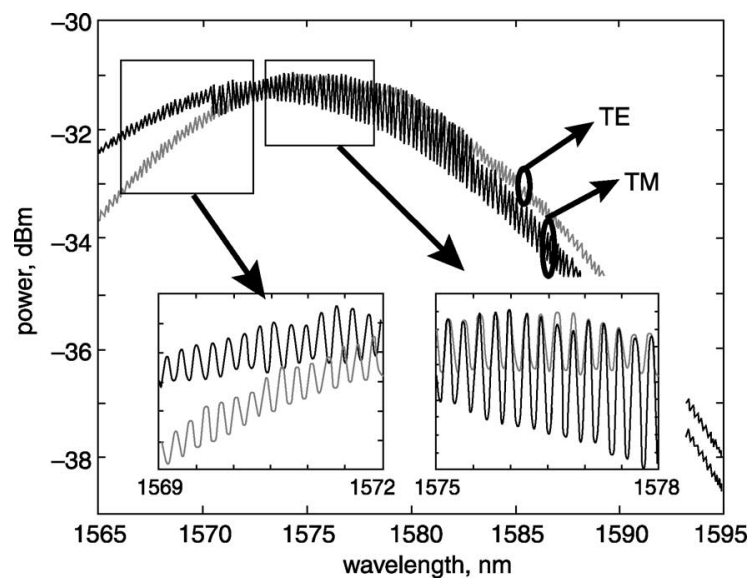

Fig. 2 Polarisation resolved spectra Inset: ripples in phase and out of phase
There is evidence of a small dip in the TE gain spectra at $1585 \mathrm{~nm}$. This arises due to a decrease in the ripple modulation depth, which later recovers. It should be noted for later reference that the TE and TM gains at $1580 \mathrm{~nm}$ under bias of $400 \mathrm{~mA}$ are equal within error on the graph, and that the spectra obtained here are dependent on the value of the reflectivity. A change in these parameters would lead to a vertical shift in the spectra obtained, but would not change their profile. Confidence in the spectra presented in Fig. 3 is justified: the peak gain at each bias current is in agreement with the single-pass gain measured as a ratio of the power out to the power in.

Birefringence has also been considered as an effect responsible for NPR $[4,13]$. To observe and quantify this effect, the refractive index is calculated for both the TE and TM modes. If there is a birefringent effect in the device then the period of the modulation ripples is different for both polarisations, as light travels at different speeds for each mode. In other words, the ripples in the ASE spectrum go in and out of phase with respect to each other, due to the small but finite difference in the periods of the ripple [14]. These effects can be seen in the insets of Fig. 2. Both modulations are in phase at $1577 \mathrm{~nm}$. At $1570 \mathrm{~nm}$, however, they are out of phase. This means that there are different refractive indices for the TE and TM modes, and that there must be birefringence in the device. The refractive index is measured using the ASE spectra recorded for the gain calculations. Using the definition of the free-spectral range in a Fabry-Perot resonator, the refractive index for both modes can be calculated:

$$
\Delta \lambda=\frac{2 n_{g, e f f} L}{k}-\frac{2 n_{g, e f f} L}{k+1}=\frac{2 n_{g, e f f} L}{k(k+1)} \approx \frac{\lambda^{2}}{2 n_{g, e f f} L}
$$

where $\lambda$ is the wavelength, $n_{g, e f f}$ is the effective group refractive index, $L$ is the length of the device and $k$ is the wavevector. Using this equation we can extract $n_{g, e f f}$ as a function of wavelength. $\Delta \lambda$ is the trough-to-trough wavelength separation, as measured from the ripples on the ASE spectra in Fig. 2, and $\lambda$ is taken as the central wavelength from trough to trough. In this way, a refractive index can be calculated for each central wavelength. Figures $4 a$ and $b$ show the effective refractive index dependence from $1560 \mathrm{~nm}$ to $1590 \mathrm{~nm}$ at $400 \mathrm{~mA}$ for the TE and TM modes, respectively. The effective refractive index is found to be 4.03 for the TE mode and 3.95 in the TM mode, where the refractive index was averaged over the complete range of wavelengths, from $1560 \mathrm{~nm}$ to $1590 \mathrm{~nm}$, providing quantitative evidence of the birefringence of the device under study. These values are consistent with previously documented results [15]. Furthermore, we can consider the dispersive nature of the semiconductor material and the dispersion parameter for each mode can be extracted using the following expression:

$$
n_{g, e f f}=n_{e f f}-\lambda \frac{d n}{d \lambda}
$$

where $n_{g, e f f}$ corresponds to the refractive index found from the free spectral range, $n_{\text {eff }}$ is effective wavelength independent refractive index and $d n / d \lambda$ the dispersive term. It is found that the scatter of the refractive index data, in Fig. 4, is too large to extract a meaningful quantitative value. However, it is clear from the slope that the dispersion parameter for the TM mode is less than that of TE, in agreement with the results from [15]. Moreover the TE refractive index is higher than the TM refractive index and this difference between refractive indices decreases slightly 

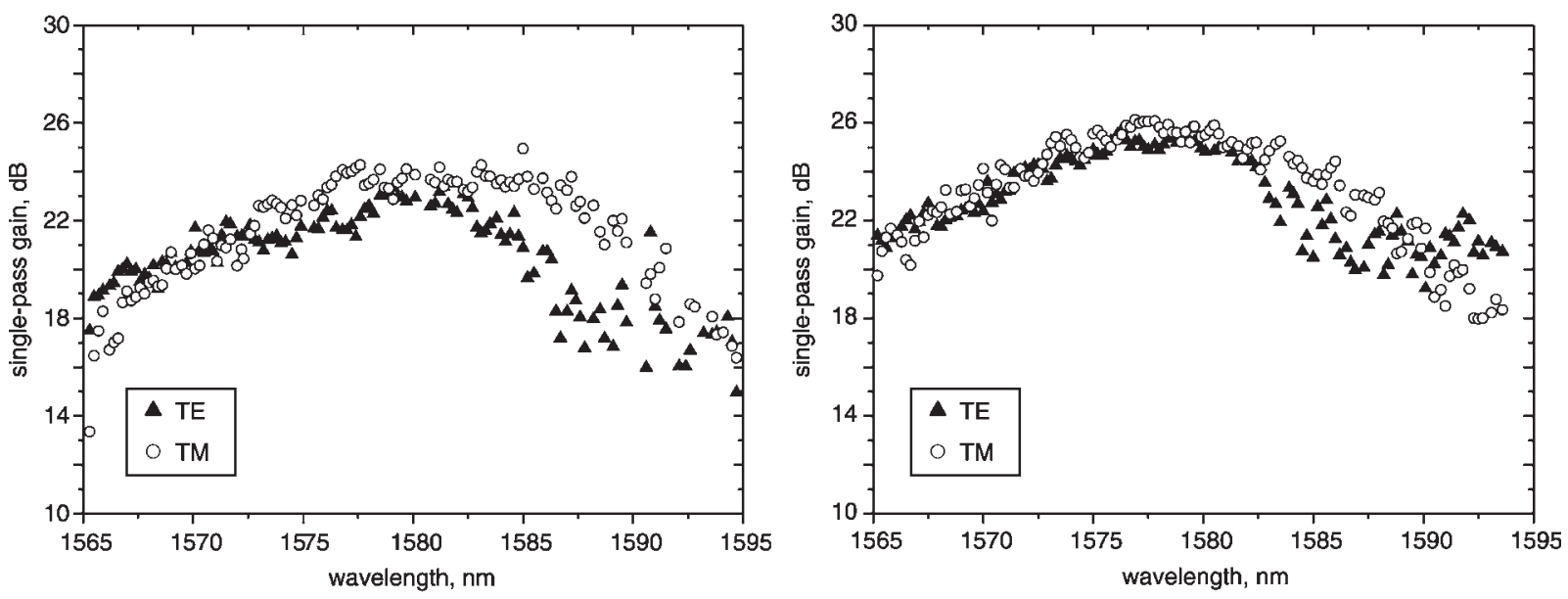

a
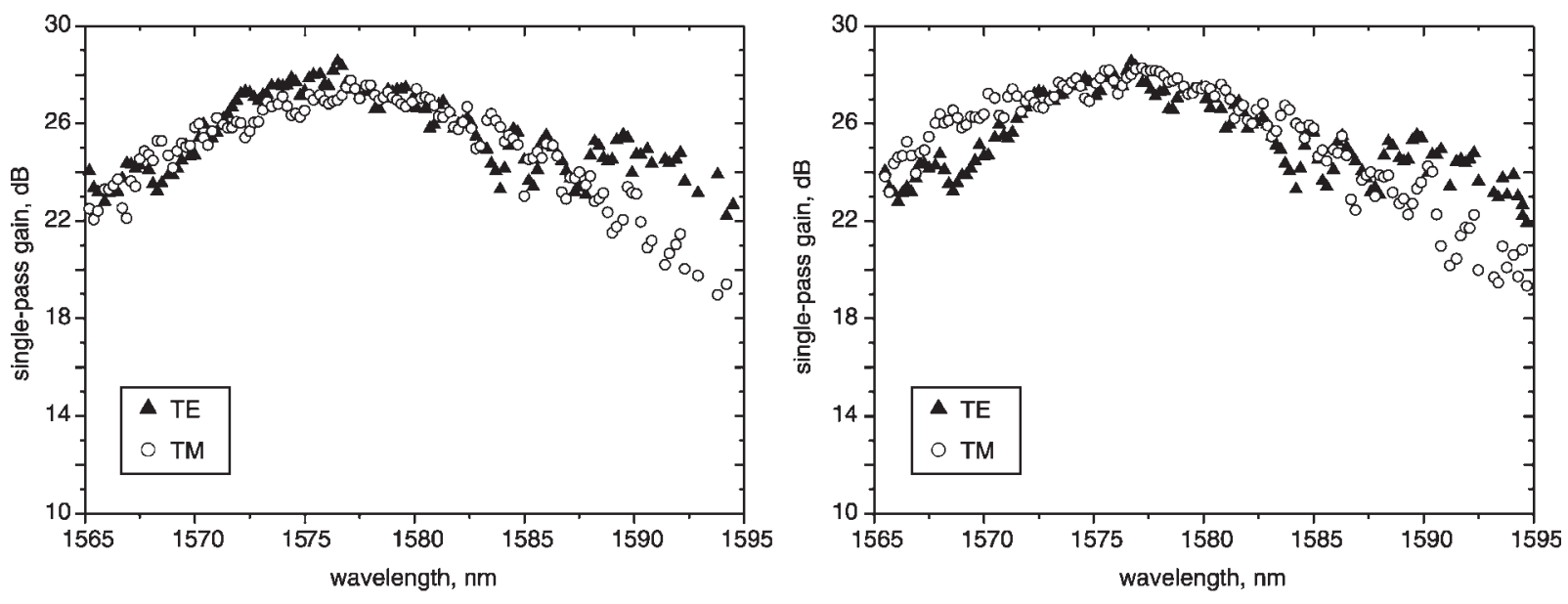

$c$

d

Fig. 3 Polarisation resolved gain spectra for $I=300 \mathrm{~mA}, 350 \mathrm{~mA}, 400 \mathrm{~mA}$ and $450 \mathrm{~mA}$

a $I_{\text {bias }}=300 \mathrm{~mA}$

$b I_{\text {bias }}=350 \mathrm{~mA}$

$c I_{\text {bias }}=400 \mathrm{~mA}$

$d I_{\text {bias }}=450 \mathrm{~mA}$

with increasing wavelength. This birefringence is a second possible origin of the NPR in the SOA.

\subsection{Gain and birefringence in SOA with injection}

The experimental results discussed so far investigate possible sources of polarisation rotation due to the underlying properties of the waveguide. To validate the earlier results and to study the effect of the gain discrepancy and birefringence on the polarisation of an injected beam, a second set of experiments is undertaken. The ellipticity of the output amplified signal is measured as a function of the input orientation and power of a linearly polarised injected signal.

Figure 5 shows the experimental set-up. The probe signal is provided by a tunable laser source. The polarisation of the free space signal is controlled by several components: (i) a polariser, to achieve a linearly polarised signal, (ii) a half-wave plate, to rotate the linearly polarised signal; and (iii) a quarter-wave plate, to compensate the mismatch between the operating wavelength of the device and the operating wavelength of the plates. The optical signal is then launched into the SOA using a $0.25 \mathrm{NA}$ AR microscope objective. The launching conditions are optimised using the two mirrors and micro-control lens mount. The ASE spectrum with $2.3 \mathrm{dBm}$ injected power and without injected power can be seen in the inset of Fig. 5. From this, we can observe a suppression of more than $6 \mathrm{~dB}$ of the ASE indicating good injection has been achieved. A band-pass filter centred at $1580 \mathrm{~nm}$ with a bandwidth of $10 \mathrm{~nm}$ is placed in front of the polarimeter to reduce the influence of the random polarisation of the ASE. The polarimeter measures the ellipticity of the output signal. The ellipticity is defined as the ratio of the length of the minor axis of the polarisation ellipse by the length of the major axis. An ellipticity of zero means that the polarisation is linear. An ellipticity of -1 corresponds to circular polarisation since both lengths are equal; the negative sign is a convention to define the left-handed rotation.

The output ellipticity as a function of input orientation for a bias current of $350 \mathrm{~mA}$ and an injection wavelength of $1580 \mathrm{~nm}$ is shown in Fig. 6. This wavelength was specifically chosen since it corresponds to the peak gain of the TE and TM modes as shown in Fig. 3. The ellipticity is recorded for injected powers of 83.3, 166, 208 and $413 \mu \mathrm{W}$. As the injection angle increases, the ellipticity decreases, reaching a minimum at $48^{\circ}$, independently of input power. A minimum of -0.94 is achieved at $48^{\circ}$ injection for $166 \mu \mathrm{W}$ of injected power. At the minimum of ellipticity curves, the output is approaching a circular polarisation. The fact that the minimum is reached at an angle greater than $45^{\circ}$ indicates that the TE gain must be greater than the TM gain. For example, at an input orientation of $48^{\circ}$, there will be more light injected into the TM mode than the TE mode and in order for the output to approach a circular polarisation the gain in TE mode must 

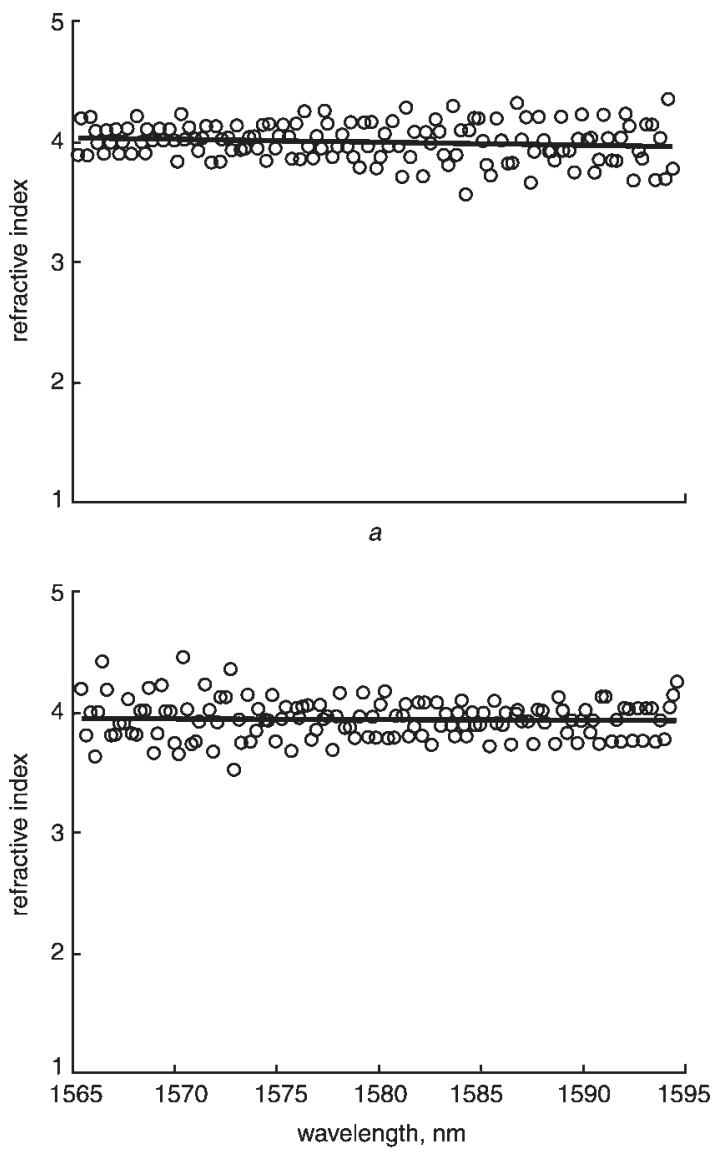

b

Fig. 4 Refractive index dependence on wavelength at $I=$ $350 \mathrm{~mA}$ for TE and TM modes

a TE mode

$b$ TM mode

compensate for the lower injected power. The negative sign shows that the output polarisation is of left-handed rotation, indicating that the TE axis is slower than the TM axis, in agreement with the result from the previous experiment with no injection. At $90^{\circ}$ the ellipticity is zero, the linear input is injected along the TM mode, propagates and is amplified along this mode. This indicates that there is no modification of the principal axes over this power range.

To develop a more quantitative understanding of the experiment, we have written a model based on a propagating electric field, $\boldsymbol{E}$, having two components aligned along the modes of the waveguide:

$$
\boldsymbol{E}=e^{i(\omega t-k z)}\left(\boldsymbol{E}_{0 T E} e^{i \phi_{T E}}+\boldsymbol{E}_{0 T M} e^{i \phi_{T M}}\right)
$$

where $\boldsymbol{E}_{0 T E(T M)}$ is the $\boldsymbol{E}$ component along the TE (TM) direction and its phase is $\phi_{T E(T M)} . \boldsymbol{E}_{T E}$ and $\boldsymbol{E}_{T M}$ are expressed as:

$$
\left\{\begin{array}{l}
\boldsymbol{E}_{0 T E}=\rho E \cos (\theta) \boldsymbol{u}_{T E} \\
\boldsymbol{E}_{0 T M}=E \sin (\theta) \boldsymbol{u}_{T M}
\end{array}\right.
$$

where $\rho$ is the ratio of the single-pass gain in the TE mode to the single-pass gain in the TM mode, $\theta$ is the input angle and $\boldsymbol{u}_{T E(T M)}$ is the unit vector along the horizontal (vertical) axis. The amplitude $E$ of the input field is taken to be unity in our model. The phases $\phi_{T E}$ and $\phi_{T M}$ are defined as follows:

$$
\phi_{T E}=\frac{2 \pi L n_{T E}}{\lambda} \text { and } \phi_{T M}=\frac{2 \pi L n_{T M}}{\lambda}
$$

As shown in Fig. 6, our numerical results fit well the experimental data using only two parameters $\rho$ and the phase difference, $\Delta \phi=\phi_{T M}-\phi_{T E}$. The ellipticity as a function of orientation is fitted using only one value of $\rho$ and $\Delta \phi$ for each injected power. Figure 7 illustrates their power dependences. Within the range of injected power, the gain ratio increases up to a value of 1.25 for an injected power of $413 \mu \mathrm{W}$, indicating that the single-pass TE gain becomes increasingly greater than the single-pass TM gain. As the injected power is decreased to zero, $\rho$ converges towards 1 , indicating that the single-pass TE gain approximates to the single-pass TM gain. This is consistent with the results of Fig. 3, where the gain spectra are obtained under conditions of no injection.

The phase difference $\Delta \phi$ is negative, demonstrating that $n_{T E}$ is larger than $n_{T M}$. As the injected power is increased, the difference between the TE and TM refractive indices increases nonlinearly. For future studies the polarimeter

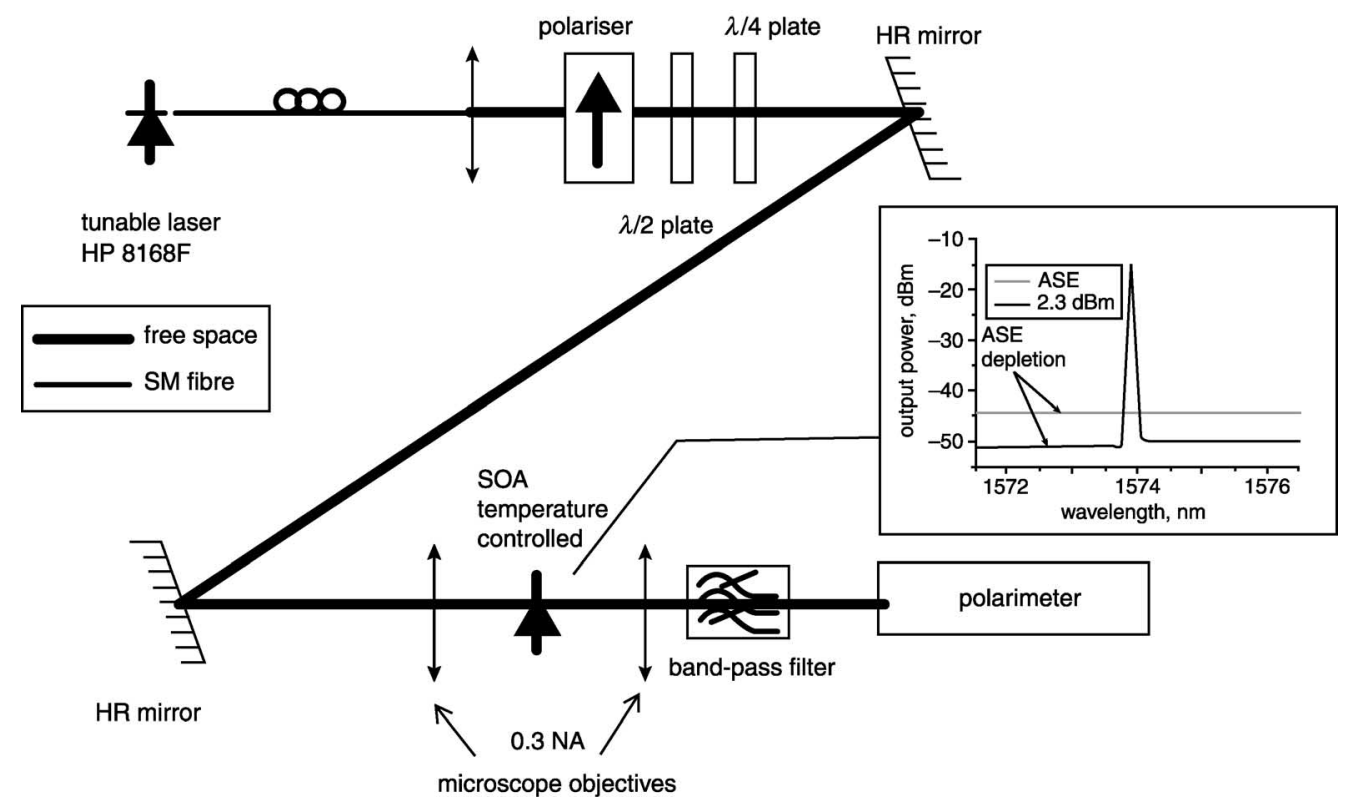

Fig. 5 Experimental set-up of injection experiment Inset: spectra of ASE without and with injection 


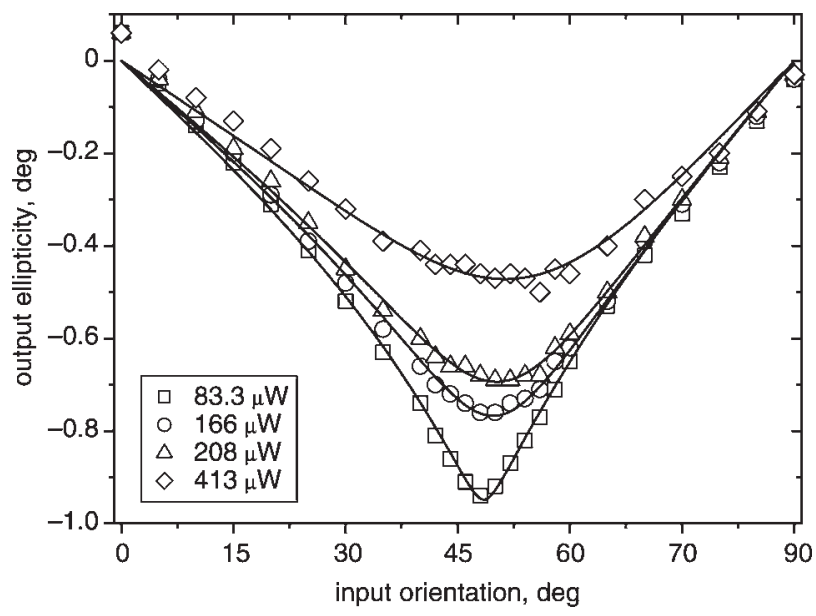

Fig. 6 Output ellipticity as a function of angle of injection of a linearly polarised input for $-10 \mathrm{dBm},-7 \mathrm{dBm},-6 \mathrm{dBm}$ and $-3 \mathrm{dBm}$

Symbols represent experimental data; simulations are represented by lines $I_{\text {bias }}=350 \mathrm{~mA} ; \lambda=1580 \mathrm{~nm}$

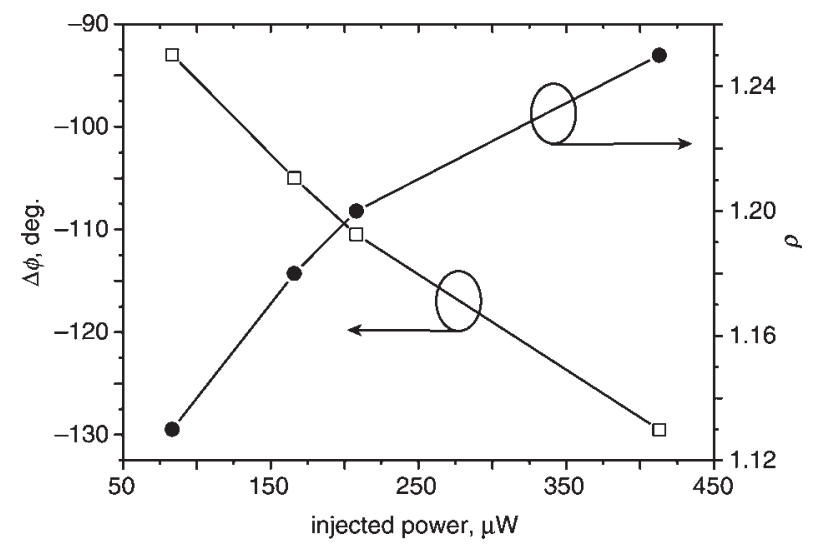

Fig. 7 Gain ratio between TE and TM modes and phase difference as a function of input power

Lines are a guide to the eye

$I_{\text {bias }}=350 \mathrm{~mA} ; \lambda=1580 \mathrm{~nm}$

data can be considered more reliable as there are no assumptions made in the fitting, unlike the facet reflectivity in the gain spectrum analysis. However, this approach is limited, since only relative gain values can be extracted. Furthermore, due to the agreement between the two methods, we can have confidence in the gain spectra of Fig. 3.

\section{Conclusions}

We investigated the origin of the nonlinear polarisation rotation in semiconductor optical amplifiers, using two techniques Two phenomena were considered as sources of the NPR: asymmetric gain between the TE and TM and birefringence. In the first experiment, there is no injected signal, allowing for the underlying properties of the waveguide to be probed. Polarisation resolved ASE spectra were recorded and the gain and refractive index for the two eigenmodes of the SOA waveguide were calculated. It was found, using this method, that a difference in the refractive indices of the two modes could be determined, with that along the TE axis being greater than that along the TM axis. Furthermore, estimations of effective refractive indices of the two modes could be extracted. The values obtained are in agreement with the literature.

A new approach has been developed to investigate the gain and refractive index differences of the two eigenmodes. The change in polarisation state of an injected signal at the output of the device is measured using a polarimeter. From the fit of the experimental results, with a basic electric field propagation model, we are able to measure the TE and TM gain ratio and the birefringence as a function of injected power. It is found that, even though the TE and TM gains are close under low optical injection, consistent with the gain extracted from the analysis of the ASE spectra, the difference in gain between the two modes increases with increasing injected power. A higher refractive index for the TE axis compared to that of the TM is confirmed, in agreement with the spectral measurements. The birefringence is also seen to increase with increasing injected power.

\section{Acknowledgment}

This work is supported by Enterprise Ireland under the Research Innovation Fund, and S. Philippe would like to thank the Irish Research Council for Science, Engineering and Technology for its support.

\section{References}

1 Asghari, M., White, I.H., and Penty, R.V.: 'Wavelength conversion using semiconductor optical amplifiers', J. Lightwave Technol., 1997, 15, (7), pp. 1181-1190

2 Pastor, D., Martinez, A., Capmany, J., Sales, S., Ortega, B., and Munoz, P.: 'Experimental characterization of XGM-SOA-based wavelength converted SCM systems', IEEE Photonics Technol. Lett., 2003, 15, (1), pp. $114-116$

3 Lee, S-L., Gong, P-M., and Yang, C-T.: 'Performance enhancement on SOA based four-wave mixing wavelength conversion using an assisted beam', IEEE Photonics Technol. Lett., 2002, 14, (12), pp. 1713-1715

4 Manning, R.J., Antonopoulos, A., Le Roux, R., and Kelly, A.E.: 'Experimental measurement of nonlinear polarization rotation in semiconductor optical amplifiers', Electron. Lett., 2001, 37, (4) pp. 229-231

5 Soto, H., Erasme, D., and Guekos, G.: 'Cross polarization modulation in semiconductor optical amplifiers', IEEE Photonics Technol. Lett., 1999, 11, pp. 970-972

6 Patrick, D.M., Ellis, A.D., Davies, D.A.O., Tatham, M.C., and Sherlock, G.: 'Demultiplexing using polarization rotation in a semiconductor laser amplifier', Electron. Lett., 1994, 30, (4), pp. 341-342

7 Stephens, M.F.C., Asghari, M., Penty, R.V., and White, I.H.: 'Demonstration of ultrafast all-optical wavelength conversion utilizing birefringence in semiconductor optical amplifiers', IEEE Photonics Technol. Lett., 1997, 9, (4), pp. 449-451

8 Liu, Y., Hill, M.T., Tangdiongga, E., de Waardt, H., Calabretta, N., Khoe, G.D., and Dorren, H.J.S.: 'Wavelength conversion using nonlinear polarization rotation in a single semiconductor optical amplifier', IEEE Photonics Technol. Lett., 2003, 15, (1), pp. 90-92

9 Dorren, H.J.S., Lenstra, D., Liu, Y., Hill, M.T., and Khoe, G.: 'Nonlinear polarization rotation in semiconductor optical amplifiers: theory and application to all-optical flip-flop memories', IEEE J. Quantum Electron., 2003, 39, (1), pp. 141-148

10 Hakki, B., and Paoli, T.: 'Gain spectra in GaAs double-heterostructure injection lasers', J. Appl. Phys., 1975, 46, (3), pp. 1299-1306

11 Meritt, S.A., Dauga, C., Fox, S., Wu, I.-F., and Dagenais, M. 'Measurement of the facet modal reflectivity spectrum in high quality semiconductor travelling wave amplifiers', J. Lightwave Technol., 1995, 13, (3), pp. 430-433

12 Guekos, G.: 'Photonic devices for telecommunications' (SpringerVerlag, 1999), p. 329

13 Soto, H., Dominguez, J.C., Erasme, D., and Guekos, G.: 'Demonstration of an all-optical switch using cross-polarization modulation in semiconductor optical amplifiers', Microw. Opt. Technol. Lett., 2001, 29, (3), pp. 205-209

14 Diez, S., Schimt, C., Ludwig, R., Weber, H.G., Doussiere, P., and Duceiller, T.: 'Effect of birefringence in bulk semiconductor optical amplifier on FWM', IEEE Photonics Technol. Lett., 1998, 10, (2), pp. $212-214$

15 Guekos, G. (Ed.): 'Photonic devices for telecommunications' (Springer-Verlag, 1999), pp. 144-145 